\title{
The real additional value of FDG-PET in detecting the occult primary tumour in patients with cervical lymph node metastases of unknown primary tumour
}

\author{
Remco de Bree
}

Published online: 9 September 2010

(C) The Author(s) 2010. This article is published with open access at Springerlink.com

Cervical lymph node metastases may be the first manifestation of cancer. In some patients presenting with a cervical lymph node metastasis, the primary tumour remains elusive after extensive diagnostic work-up. In such cases, the patient is classified as having a so-called unknown primary tumour (UPT). The incidence of UPT ranges from 3 to $7 \%$ of all head and neck cancers. The most commonly histological diagnosis is squamous cell carcinoma (SCC). In case of SCC, the following diagnostic procedures are performed: physical head and neck examination at the outpatient clinic, CT or MRI of the head and neck area, X-ray or CT of the chest, and endoscopy under general anaesthesia of the whole upper aerodigestive tract (panendoscopy). During the panendoscopy, directed biopsies are taken from the nasopharynx, the base of the tongue and the tonsils (or tonsillectomy). Unfortunately, the yield of these directed biopsies taken during panendoscopy is low. Consequently, a substantial number of UPTs remains undetected with the conventional diagnostic procedures. In case of UPT, treatment mostly consists of a (modified) radical neck dissection in combination with irradiation of the whole pharyngeal axis. The irradiation of this large surface area aims the elimination of a probably very small (at least undetectable) malignant primary tumour. Unfortunately, this radiation treatment may cause considerable iatrogenic morbidity, e.g. mucositis, xerostomia, mucosal atrophy and even osteoradionecrosis.

R. de Bree $(\square)$

Department of Otolaryngology/Head and Neck Surgery,

VU University Medical Center, De Boelelaan 1117,

1081 HV Amsterdam, PO Box 7057,

1007 MB Amsterdam, The Netherlands

e-mail: r.bree@vumc.nl
All the diagnostic procedures mentioned are performed because the detection of the primary tumour is important. Detection of the primary tumour generally allows for a less extensive treatment (e.g. smaller radiation field). It may lead to a more specific therapy which in its turn will give a more appropriate treatment, resulting in an improved locoregional control and lower morbidity.

Several studies have examined the value of ${ }^{18}$ FDG-PET for detecting UPT. In a comprehensive review of Rusthoven et al. [1] on the use of ${ }^{18}$ FDG-PET for the detection of UPT which comprises 16 studies with 302 patients, a sensitivity of $88.3 \%$, specificity of $74.9 \%$ and accuracy of $78.8 \%$ are observed. Similar results were demonstrated in more recent studies (PET and PET/CT) and the study published in this issue [2, 3]. A problem with interpretation and comparison of these studies is their lack of uniformity in the inclusion criteria: after physical examination only or after diagnostic imaging by CT and/or MRI and/or endoscopic examination under general anaesthesia with taking of directed biopsies of areas most likely to harbour an occult primary tumour. Thus, the definition of UPT differs among studies. It may be obvious that the chance of detecting the occult primary tumour is higher in patients in whom the primary tumour is not found after physical examination only than in patients who underwent an extensive diagnostic work-up, including diagnostic imaging. To investigate the additional value of FDG-PET, only patients with UPT after complete conventional work-up should be included as in the study published in the present issue of European Archives of Otorhinolaryngology and Head \& Neck [3].

Moreover, the definition of sensitivity differs between studies. Most studies used the combined detection results of all diagnostic techniques during follow-up as reference standard. In principle, a primary tumour should be present 
in all patients (except if spontaneous complete regression is believed), but that in some patients these tumours will not be detected or become manifest due to wide field irradiation. Therefore, all undetected primary tumours in those patients should be considered as false negative. Consequently, the real sensitivity is much lower than the reported sensitivity (70-100\%) [1-4]. The detection rate of occult primary tumours is probably a more realistic outcome parameter than sensitivity. Rusthoven et al. [1] found a detection rate by FDG-PET in $24.5 \%$ of patients.

Another aspect is that specificity remains too low because of too many false-positive results. The tonsils and the base of tongue appeared to be the localizations with the highest rate of false-positive findings [1]. This is probably caused by some asymmetric physiological uptake. It may be difficult to differentiate between physiological and pathological uptake in the head and neck area. It is important to know the specific sites with physiological uptake in detail when reading FDG-PET scans of the head and neck [5].

Miller et al. [8] showed in a prospective study $(n=31)$ that the combination of ${ }^{18}$ FDG-PET and a panendoscopy with biopsies detected $45.2 \%$ (14/31) of UPT. Of these 14 cases, 5 patients had a negative PET, but a positive finding by panendoscopy and biopsies. Therefore, a negative PET does not preclude the need for panendoscopy with biopsy to detect the UPT. In addition, they concluded that the overall risk of subsequent manifestation of a primary tumour of the upper aerodigestive tract was very low $(5.8 \%)$ in patients that have had a negative PET and a negative panendoscopy with biopsy, demonstrating the efficacy of this combined approach [6].

In order to decrease false-positive and false-negative findings, FDG-PET uptake was quantified using standardized uptake value (SUV, i.e. the ratio of measured uptake in a static scan obtained 60 min after FDG injection over the injected dose and normalized for volume of distribution, e.g. bodyweight). PET quantification with SUVs is affected by many technical and physiological factors. As a result, differences in FDG-PET study methods hamper the determination of universal guidelines for interpretation of FDG uptake. These differences concern patient factors (e.g. plasma glucose levels), time interval between injection and scanning, image reconstruction technique, image quality and SUV measure used. To improve the usefulness of SUVs, calibration of PET scanners and standardization of PET-scanning protocols should be performed. Guidelines for standardization and quantification of FDG uptake have been developed [7]. Moreover, normal SUV values of FDG uptake at sites with physiological FDG uptake should be available to improve distinguishing physiological from pathological FDG uptake at specific sites.
An important methodological problem is how to determine the specific contribution of each diagnostic technique. If, for example, an occult primary tumour in the tonsil is detected by FDG-PET, it can be anticipated that this primary tonsillar carcinoma would also have been detected by tonsillectomy which is part of the conventional (non-FDGPET based) work-up. In such a scenario, the additional value of FDG-PET is questionable.

The most accurate study design to examine the real additional value of FDG-PET has been used in a prospective, multicentre study in patients with a UPT in which physical examination, indirect laryngoscopy and CT/MRI had not revealed the occult primary. All patients underwent a blinded FDG-PET and a panendoscopy with directed biopsies. After the panendoscopy, but in the same narcosis session, the result of the FDG-PET was communicated to the surgeon, and additional biopsies could be taken based on these findings. In this Dutch ZonMw-funded multicentre PRIMUS study, the additional value of FDG-PET was examined by comparing the conventional standard work-up with a FDG-PET-based work-up. In this way, the real additional value of PET-guided biopsies can be determined. Unfortunately, no results have been published yet.

If FDG-PET is considered to be of real additional value in detecting occult primary tumours in patients with cervical lymph node metastases of an unknown primary tumour, the question arises when to perform a FDG-PET scan. Frequently, patients presenting with cervical lymph node metastases as first manifestation of cancer have extensive disease of the neck and are at risk for distant metastases [8]. Besides, primary tumours outside the head and neck area can metastasize to the neck. In case of distant metastases or primary tumours below the clavicles, imaging of the head and neck area and panendoscopy can be avoided. Another reason to perform FDG-PET upfront may be waiting time for FDG-PET or other logistic problems. On the other hand, if the occult primary tumour is detected by conventional imaging techniques, FDG-PET is not needed. PET is not without drawbacks, not at least its availability, costs, radiation exposure, detection of incidentalomas and burden to the patient.

Detection of only about $25 \%$ of unknown primary tumours by FDG-PET does not seem very impressive, but for the individual patient this means a less extensive treatment possibly leading to a substantial reduction of morbidity. FDG-PET should be performed in HNSCC patients presenting with UPT. However, the real additional value of FDG-PET has still to be defined. Therefore, a large multicentre study using aforementioned study design in a homogeneous (squamous cell carcinoma only) group of patients with UPT after physical examination and CT or MRI should be performed. 
Open Access This article is distributed under the terms of the Creative Commons Attribution Noncommercial License which permits any noncommercial use, distribution, and reproduction in any medium, provided the original author(s) and source are credited.

\section{References}

1. Rusthoven KE, Koshy M, Paulino AC (2004) The role of fuorodeoxyglucose positron emission tomography in cervical lymph nodes metastases from unknown primary tumor. Cancer 101:2642-2649

2. Padovani D, Aimoni C, Zucchetta P, Paluzzi A, Pastore A (2009) 18 -FDG PET in the diagnosis of laterocervical metastases from occult carcinoma. Eur Arch Otorhinolaryngol 266:267-271

3. Yabuki K, Tsukuda M, Horiuchi C, Taguchi T, Nishimura G (2010) Role of ${ }^{18}$ F-FDG PET in detecting primary site in the patient with primary unknown carcinoma. Eur Arch Otorhinolaryngol. doi: 10.1007/s00405-010-1371-3
4. Regelink G, Brouwer J, De Bree R et al (2002) Detection of unknown primary tumours and distant metastases in patients with cervical metastases: value of FDG-PET versus conventional modalities. Eur J Nucl Med 29:1024-1030

5. Burrel SC, Van den Abbeele AD (2005) 2-Deoxy-2-[F-18]fluoroD-glucose-positron emission tomography of the head and neck: an atlas of normal uptake and variants. Mol Imaging Biol 7:244-256

6. Miller FR, Karnad AB, Eng T, Hussey DH, Stan McGuff H, Otto RA (2008) Management of the unknown primary carcinoma: longterm follow-up on a negative PET scan and negative panendoscopy. Head Neck 30:28-34

7. Boellaard R (2009) Standards for PET image acquisition and quantitative data analysis. J Nucl Med 50:11s-20s

8. Senft A, de Bree R, Hoekstra OS et al (2008) Screening for distant metastases in head and neck cancer patients by chest CT or whole body FDG-PET: a prospective multicenter trial study. Radiother Oncol 87:221-229 